HETEROCYCLES, Vol. , No. , , pp. -. (c) The Japan Institute of Heterocyclic Chemistry

Received, , Accepted, , Published online, COM-06-(Please do not delete.

\title{
A CONCISE SYNTHESIS OF SOLAMIN AND CIS-SOLAMIN, MONO-THF ACETOGENINS FROM ANNONA MURICATA
}

\author{
Hidefumi Makabe, ${ }^{* a, b}$ Asuka Kuwabara, ${ }^{\text {a }}$ Yasunao Hattori, ${ }^{\text {b,c }}$ and Hiroyuki \\ Konno $^{\text {d }}$
}

${ }^{\mathrm{a}}$ Sciences of Functional Foods, Graduate School of Agriculture, Shinshu University, 8304 Minami-minowa, Kami-ina, Nagano 399-4598, Japan

${ }^{\mathrm{b}}$ Interdisciplinary Graduate School of Science and Technology, Shinshu University, 8304 Minami-minowa, Kami-ina, Nagano 399-4598, Japan

${ }^{\mathrm{c}}$ Satellite Venture Business Laboratory, Shinshu University, 3-15-1 Tokida, Ueda, Nagano 386-8567, Japan

${ }^{\mathrm{d}}$ Department of Chemistry, Graduate School of Medicinal Science, Kyoto

Prefectural University of Medicine, Kyoto 603-8334, Japan

*E-mail address: makabeh@shinshu-u.ac.jp

\begin{abstract}
A concise total synthesis of solamin (1) and cis-solamin (2) was performed using an epoxy alcohol (11) as a versatile chiral building block for synthesizing the stereoisomers of the mono-THF annonaceous acetogenins.
\end{abstract}

The annonaceous acetogenins, which are endemic to certain plants of the Annonaceae, are of much interest especially due to their unique structural features and significant biological activities such as cytotoxic, antitumoral, pesticidal, antiinfective, and antifeedant ones. Biochemical studies have shown that acetogenins are the most potent inhibitors of the mitochondrial respiratory enzyme complex I (NADH-ubiquinone oxidoreductase). More than 430 compounds belonging to this family have been isolated to date, and most of them possess tetrahydrofuran (THF) or tetrahydropyran (THP) rings, hydroxyl, keto and epoxy group, double and triple bonds together with a terminal $\alpha, \beta$-unsaturated $\gamma$-lactone unit on a C-35 or C-37 long carbon chain. ${ }^{1}$ Consequently, significant effort has been devoted toward the total synthesis of annonaceous acetogenins. ${ }^{2}$ Solamin (1) is a mono-THF acetogenin, isolated from Annona muricata in $1991 .^{3}$ cis-Solamin (2) is a diastereomer of $\mathbf{1}$ and it was isolated in 1998 from same plants. ${ }^{4}$ Several syntheses of $\mathbf{1}$ and $\mathbf{2}$ were reported including our previous syntheses. ${ }^{5,6}$ In our 
previous syntheses, it was necessary to get rid of undesired diastereomer of the THF part. As to the synthesis of $\mathbf{1}$, low stereoselectivity of the epoxidation of $\mathbf{3}$ and the difficulty to separate $\mathbf{4 a}$ and $\mathbf{4 b}$ caused serious problem. To separate undesired product $\mathbf{4 b}$, benzoylation of the hydroxyl group followed by careful purification with preparative TLC was necessary. ${ }^{5 \mathrm{c}}$ For the synthesis of $\mathbf{2}$, although the stereoselectivity to construct ent-4b was improved using TBHP in the presence of $\mathrm{VO}(\mathrm{acac})_{2}$ as a catalyst, it was still necessary to introduce benzoyl group for careful separation. ${ }^{6 a}$ Thus it was difficult to synthesize mono-THF moiety in large scale (Scheme 1).

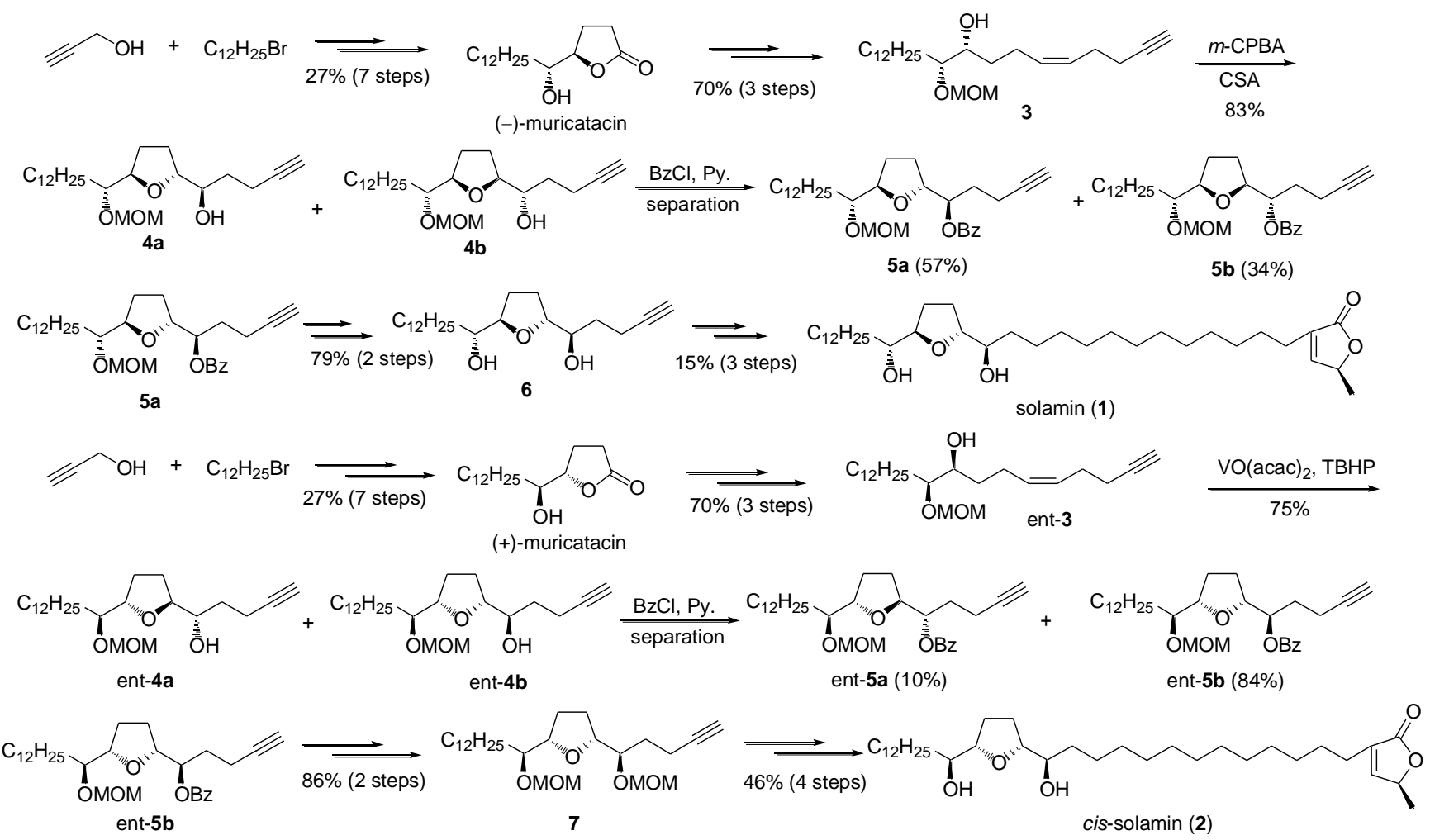

Scheme 1. Previous synthesis of solamin (1) and cis-solamin (2).

To solve these problems, we have developed a concise synthesis of solamin (1) and cis-solamin (2) using systematic and stereoselective construction of the mono-THF moieties without separating undesired diastereomers from chiral epoxy alcohol 11. ${ }^{8}$ The synthetic strategy of $\mathbf{1}$ and $\mathbf{2}$ is shown in Scheme 3. The mono-THF moieties were synthesized from epoxy alcohol 11 using Sharpless AD mix $\beta$ for threo-trans-threo moiety 8 and AD mix $\alpha$ for threo-cis-threo THF moiety 9 (Scheme 2). 


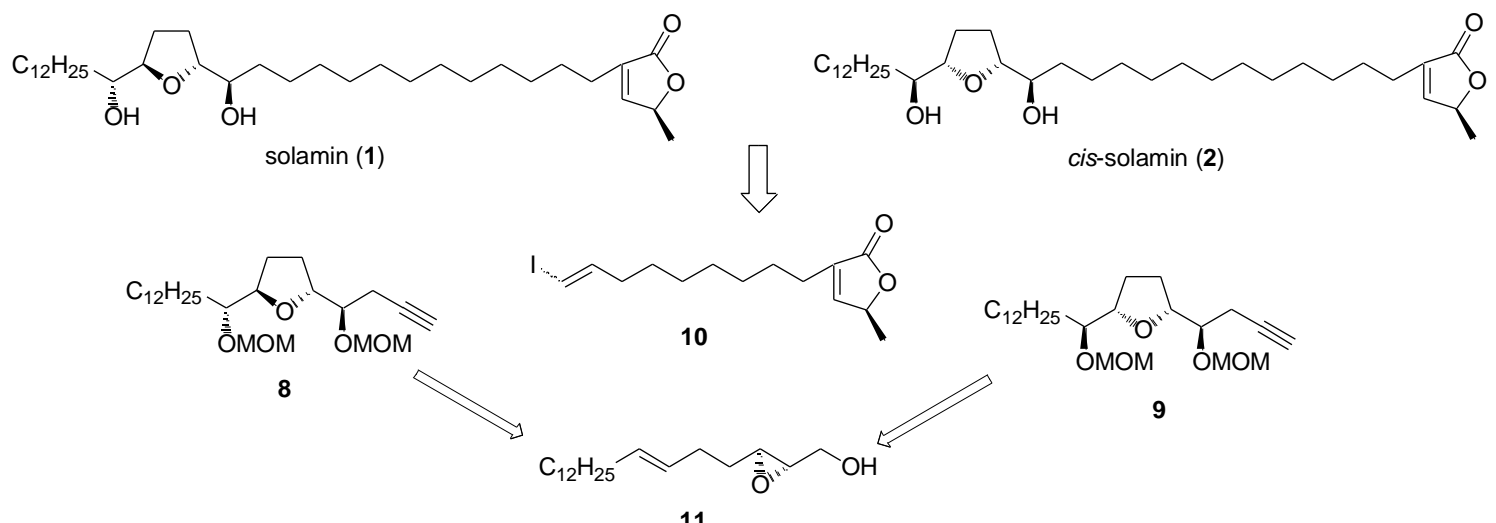

Scheme 2. Synthetic strategy of $\mathbf{1}$ and 2.

As shown in Scheme 3, the THF part 8 and $\mathbf{9}$ were synthesized from acrolein and laurylmagnesium bromide as we prepared before through 8 steps in 50 and $52 \%$ yield, respectively (Scheme 3). ${ }^{7,8}$

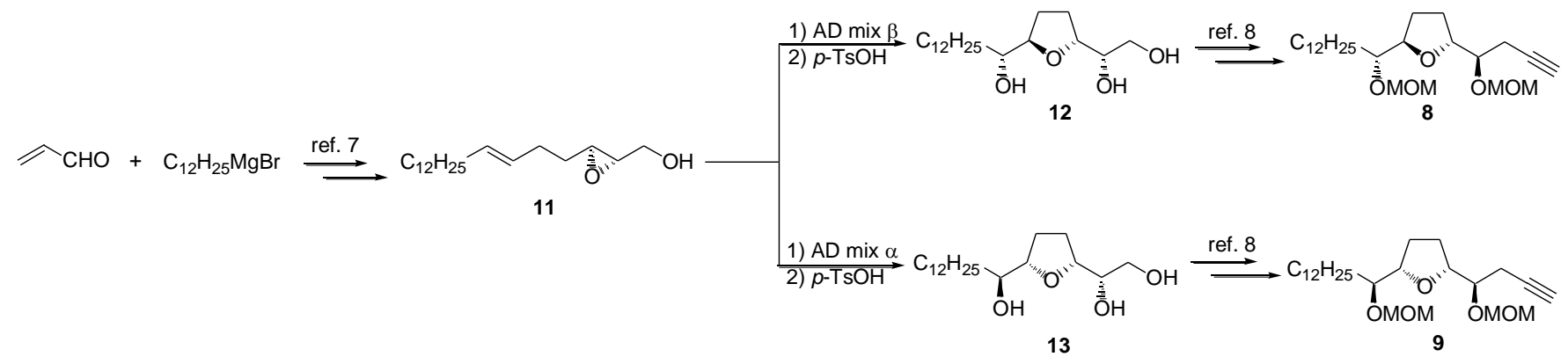

Scheme 3. Synthesis of the tetrahydrofuran moieties of $\mathbf{1}$ and 2.

The $\alpha, \beta$-unsaturated $\gamma$-lactone segment was synthesized as shown in Scheme 3. We selected vinyl iodide $\mathbf{1 4}$ as the coupling partner. Vinyl iodide $\mathbf{1 4}$ was derived from 1-bromohexane and propargyl alcohol as we reported before. ${ }^{5 \mathrm{c}}$ We examined the alkylation of $\gamma$-lactone $\mathbf{1 5}$ (prepared by the method of White et al. ${ }^{9}$ ) with diiodide 14 using LDA, LHMDS, NHMDS, and KHMDS as a base. In the case of LDA and/or LHMDS, the yield of product was very poor. On the other hand, NHMDS and KHMDS resulted in moderate yield. The counter cation of the base was extremely important for the alkylation of 15 . Oxidation with $m$ CPBA following thermal elimination of sulfoxide gave $\gamma$-lactone moiety $\mathbf{1 0}$ (Scheme 4).

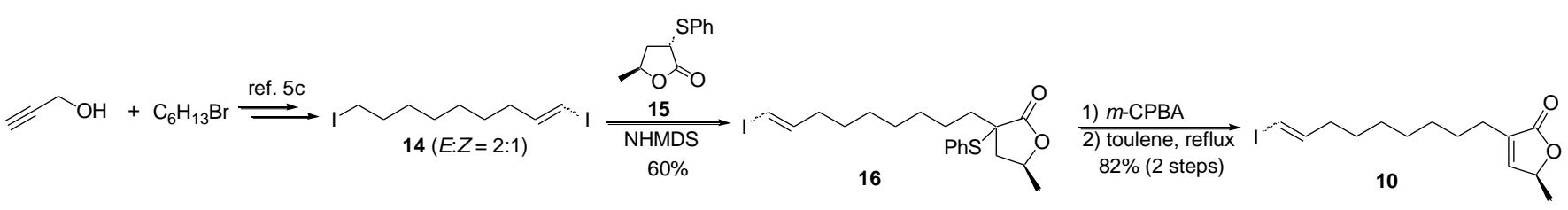

Scheme 4. Synthesis of $\gamma$-lactone moiety 5.

The THF moiety $\mathbf{8}$ and $\gamma$-lactone $\mathbf{1 0}$ was coupled by the Sonogashira cross-coupling reaction to give $\mathbf{1 7}$ in $78 \%$ yield. Diimide reduction with $p-\mathrm{TsNHNH}_{2}$ and $\mathrm{AcONa}$ in ethylene glycol diethyl ether under reflux 
afforded saturated product 18. Finally, deprotection of the MOM-protected ethers with $\mathrm{BF}_{3} \cdot \mathrm{Et}_{2} \mathrm{O}$ afforded $\mathbf{1}$ in $72 \%$ yield. The synthesis of $\mathbf{2}$ was also carried out as described for $\mathbf{1}$. The spectral and physical data of the synthetic $\mathbf{1}$ and $\mathbf{2}$ were in good agreement of those of reported values (Scheme 5). ${ }^{3,4}$

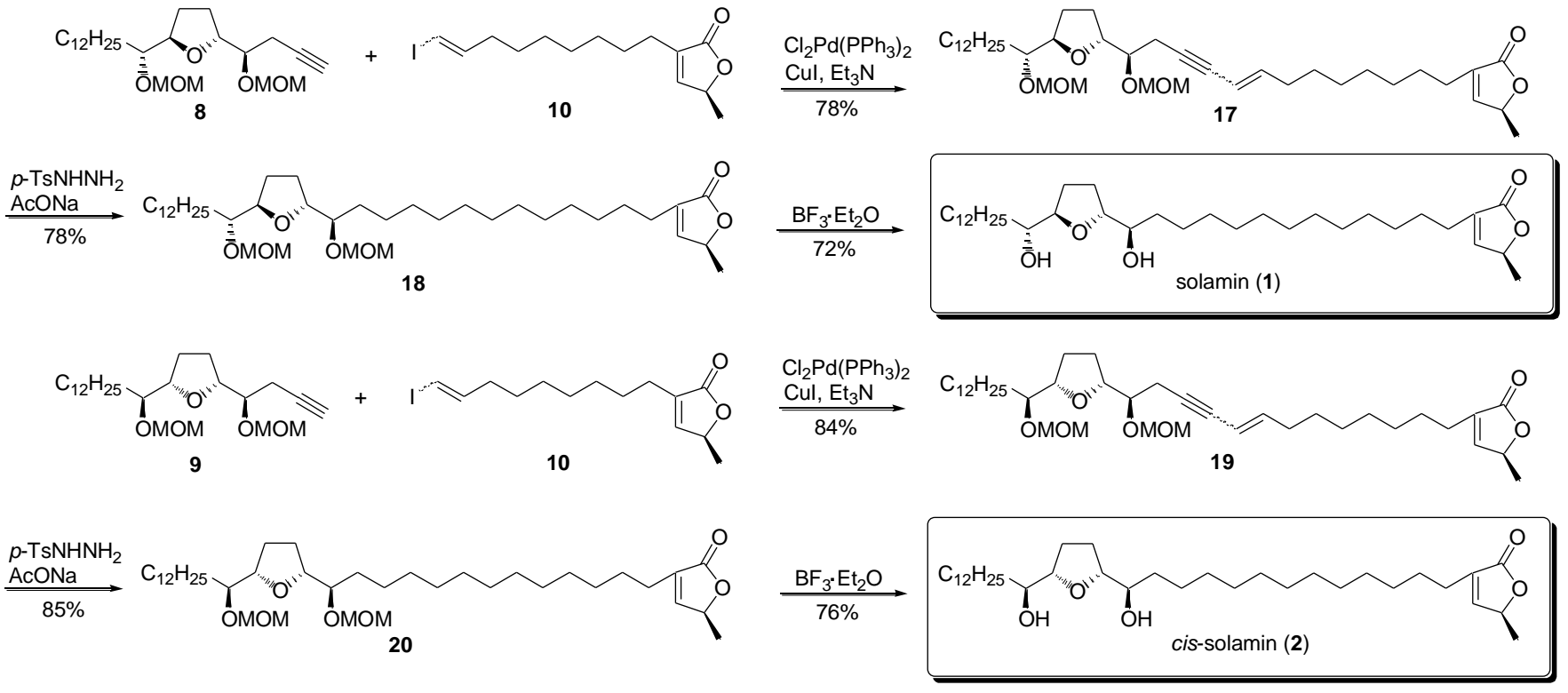

Scheme 5. Synthesis of solamin (1) and cis-solamin (2).

In conclusion, a concise synthesis of 1 and 2 was accomplished through 18 steps in $15 \%$ and $18 \%$, respectively. The overall yields of $\mathbf{1}$ and $\mathbf{2}$ were much higher than those of our previous synthesis (19 steps, $0.86 \%$ yield for $1,^{5 \mathrm{c}} 20$ steps, $3.5 \%$ for $\mathbf{2}^{6 \mathrm{a}, 6 \mathrm{~b}}$ ).

\section{EXPERIMENTAL}

General. All melting points were uncorrected. ${ }^{1} \mathrm{H}$ and ${ }^{13} \mathrm{C}$ NMR spectra were measured with a Bruker Avance DRX 500 FT-NMR spectrometer in $\mathrm{CDCl}_{3}$ at 500 and $125 \mathrm{MHz}$, respectively. Chemical shifts were relative to tetramethylsilane as an internal standard. The coupling constants were given in Hz. Mass spectra were obtained on JEOL JMS-700 mass spectrometer. IR spectra were recorded with JASCO FT-IR 480 Plus infrared spectrometer. Optical rotations were determined with a JASCO DIP-1000 polarimeter.

(8'EZ)-3-(Iodonon-9'-enyl)-5-methyl-2,5-dihydrofuran-2-one (10). To an ice-cooled solution of compound 15 (200 mg, $1.0 \mathrm{mmol})$ in THF (5 ml) was added sodium bis(trimethylsilyl)amide $(1.0 \mathrm{M}$ solution in THF, $1.0 \mathrm{ml})$. After the mixture had been stirred for $30 \mathrm{~min}$ at $0^{\circ} \mathrm{C}$, the diiodide $14(396 \mathrm{mg}$, $1.0 \mathrm{mmol})$ in HMPA $(2 \mathrm{ml})$ was added to it and the whole was allowed to warm to room temperature. The 
reaction mixture was then poured into saturated aqueous $\mathrm{NH}_{4} \mathrm{Cl}(5 \mathrm{ml})$, and the mixture was extracted with diethyl ether. The organic phase was washed with brine, dried over anhydrous $\mathrm{MgSO}_{4}$, filtered and concentrated. The residue was roughly purified with silica gel column chromatography (hexane/AcOEt $=$ 8:1) to afford $16(274 \mathrm{mg}, 60 \%)$ as a colorless oil. This compound was used for the next step without further purification. Compound $16(114 \mathrm{mg}, 0.25 \mathrm{mmol})$ was dissolved in $\mathrm{CH}_{2} \mathrm{Cl}_{2}(5 \mathrm{ml})$ and $m$-CPBA $(65 \%, 66 \mathrm{mg}, 0.25 \mathrm{mmol})$ was added at $0^{\circ} \mathrm{C}$. After the mixture was stirred for $20 \mathrm{~min}$ at this temperature, saturated aqueous $\mathrm{Na}_{2} \mathrm{~S}_{2} \mathrm{O}_{3}$ and $\mathrm{NaHCO}_{3}(1: 1,5 \mathrm{ml})$ were added. The mixture was stirred for $1 \mathrm{~h}$ and extracted with diethyl ether. The organic phase was washed with brine, dried over anhydrous $\mathrm{MgSO}_{4}$, filtered and concentrated. The residue was purified with preparative TLC (toluene/AcOEt $=15: 1$ ) to afford 10 (71 mg, 82\%) as a colorless oil. IR(film) $v_{\max }:$ 3073, 2978, 2927, 2854, 1752, 1653, 1607, 1453, 1318, 1199, 1118, 1100, 1085, 1069, 1026, 949, $860 \mathrm{~cm}^{-1} .{ }^{1} \mathrm{H}$ NMR (500 MHz, $\left.\mathrm{CDCl}_{3}\right) \delta: 1.20-1.55(10 \mathrm{H}$, m), $1.41(3 \mathrm{H}, \mathrm{d}, J=7.0 \mathrm{~Hz}), 2.05-2.20(2 \mathrm{H}, \mathrm{m}), 2.26(2 \mathrm{H}, \mathrm{t}, J=7.0 \mathrm{~Hz}), 5.00(1 \mathrm{H}, \mathrm{qd}, J=7.0,1.5 \mathrm{~Hz})$, $5.98(0.67 \mathrm{H}, \mathrm{dt}, J=15.8,1.5 \mathrm{~Hz}), 6.18(0.67 \mathrm{H}, \mathrm{m}), 6.50(0.67 \mathrm{H}, \mathrm{dt}, J=15.8,6.8 \mathrm{~Hz}), 7.00(1 \mathrm{H}, \mathrm{d}, J=1.5$ $\mathrm{Hz}) .{ }^{13} \mathrm{C}$ NMR $\left(125 \mathrm{MHz}, \mathrm{CDCl}_{3}\right) \delta: 19.19,25.11,27.30,27.33,27.83,28.24,28.35,28.54,28.71,28.89$, 28.97, 28.99, 29.02, 29.03, 29.05, 29.62, 34.59, 35.95, 74.35, 77.39, 82.23, 134.20, 141.32, 146.63, 148.90, 173.84 ppm. HREIMS Calcd for $\mathrm{C}_{14} \mathrm{H}_{21} \mathrm{IO}_{2}[\mathrm{M}]^{+}$348.0586, found 348.0590.

\section{$\left(1{ }^{\prime \prime}, R, 2\right.$ ' $R, 5 S, 5$ ' $R, 13$ ' $\left.R\right)-3-\{13$ '-Methoxymethoxy-13'-[5"-(1'"'-methoxymethoxytridecyl)tetrahydr-} ofuran-2"-yl]tetradec-8'en-10'-ynyl\}-5-methyl-2,5-dihydrofuran-2-one (17). $\left.\mathrm{Cl}_{2} \mathrm{Pd}_{(\mathrm{PPh}}\right)_{2}(2.5 \mathrm{mg}$, $3.5 \mu \mathrm{mol})$ was added to a solution of $\mathbf{1 0}(25 \mathrm{mg}, 0.07 \mathrm{mmol})$ in $\mathrm{Et}_{3} \mathrm{~N}(0.15 \mathrm{ml})$. After the solution had been stirred for $30 \mathrm{~min}$, a solution of $8(30 \mathrm{mg}, 0.07 \mathrm{mmol})$ in $\mathrm{Et}_{3} \mathrm{~N}(0.15 \mathrm{ml})$ and $\mathrm{CuI}(1.5 \mathrm{mg}, 8 \mu \mathrm{mol})$ were added. After the mixture had been stirred for $12 \mathrm{~h}$, the reaction was quenched with saturated aqueous $\mathrm{NH}_{4} \mathrm{Cl}(3 \mathrm{ml})$, and the mixture was extracted with diethyl ether. The organic phase was washed with brine, dried over anhydrous $\mathrm{MgSO}_{4}$, filtered and concentrated. The residue was purified with preparative TLC (toluene/AcOEt $=10: 1)$ to afford $17(35 \mathrm{mg}, 78 \%)$ as a colorless oil. IR(film) $v_{\max }$ : 3052, 2924, 2853, 1758, 1654, 1465, 1318, 1205, 1150, 1102, 1035, 955, $918 \mathrm{~cm}^{-1} .{ }^{1} \mathrm{H}_{\mathrm{NMR}}\left(500 \mathrm{MHz}, \mathrm{CDCl}_{3}\right)$ $\delta: 0.88(3 \mathrm{H}, \mathrm{t}, J=6.8 \mathrm{~Hz}), 1.25-1.80(34 \mathrm{H}, \mathrm{m}), 1.41(3 \mathrm{H}, \mathrm{d}, J=6.8 \mathrm{~Hz}), 1.95-2.10(4 \mathrm{H}, \mathrm{m}), 2.26(2 \mathrm{H}, \mathrm{t}, J$ $=7.2 \mathrm{~Hz}), 2.45-2.65(2 \mathrm{H}, \mathrm{m}), 3.38(3 \mathrm{H}, \mathrm{s}), 3.39(2 \mathrm{H}, \mathrm{s}), 3.40(1 \mathrm{H}, \mathrm{s}), 3.46(1 \mathrm{H}, \mathrm{m}), 3.64(1 \mathrm{H}, \mathrm{m}), 3.98$ $(1 \mathrm{H}, \mathrm{m}), 4.12(1 \mathrm{H}, \mathrm{m}), 4.67(1 \mathrm{H}, \mathrm{d}, J=6.8 \mathrm{~Hz}), 4.73-4.78(2 \mathrm{H}, \mathrm{m}), 4.84(1 \mathrm{H}, \mathrm{m}), 5.00(1 \mathrm{H}, \mathrm{qd}, J=6.8$, $1.5 \mathrm{~Hz}), 5.42(0.67 \mathrm{H}, \mathrm{dd}, J=15.8,1.8 \mathrm{~Hz}), 5.81(0.67 \mathrm{H}, \mathrm{m}), 6.03(0.67 \mathrm{H}, \mathrm{dt}, J=15.8,7.4 \mathrm{~Hz}), 6.99(1 \mathrm{H}$, $\mathrm{d}, J=1.5 \mathrm{~Hz}) \mathrm{ppm} .{ }^{13} \mathrm{C}$ NMR $\left(125 \mathrm{MHz}, \mathrm{CDCl}_{3}\right) \delta: 14.11,19.19,22.42,22.50,22.66,25.12,25.46,25.49$, $27.33,28.03,28.10,28.44,28.69,28.73,28.92,29.07,29.33,29.60,29.62,29.65,29.79,31.16,31.89$, 55.77, 66.64, 74.03, 77.65, 79.56, 80.09, 80.21, 81.70, 81.79, 96.37, 96.47, 96.65, 109.64, 134.21, 143.77, 148.89, 173.89 pm. HRFABMS Calcd for $\mathrm{C}_{39} \mathrm{H}_{67} \mathrm{O}_{7}[\mathrm{M}+\mathrm{H}]^{+}$647.4886, found 647.4890. 
$\left(1{ }^{\prime \prime}, R, 2\right.$ ' $R, 5 S, 5$ " $\left.R, 13 ' R\right)-3-\{13$ '-Methoxymethoxy-13'-[5"-(1'"'-methoxymethoxytridecyl)tetrahydrofuran-2"-yl]tetradecyl\}-5-methyl-2,5-dihydrofuran-2-one (18). A solution of sodium acetate (371 mg, $4.52 \mathrm{mmol})$ in $\mathrm{H}_{2} \mathrm{O}(12 \mathrm{ml})$ was added to a solution of 17 (16 $\left.\mathrm{mg}, 0.025 \mathrm{mmol}\right)$ and p-toluenesulfonylhydrazide $(733 \mathrm{mg}, 3.73 \mathrm{mmol})$ in diethoxyethane $(8 \mathrm{ml})$ over $5 \mathrm{~h}$. After being cooled to room temperature, the mixture was extracted with diethyl ether. The organic phase was washed with brine, dried over anhydrous $\mathrm{MgSO}_{4}$, filtered and concentrated. The residue was purified with preparative TLC (hexane/AcOEt $=4: 1)$ to afford $18\left(13 \mathrm{mg}, 78 \%\right.$ ) as a colorless oil. $[\alpha]_{\mathrm{D}}^{25}+28\left(c 0.30, \mathrm{CHCl}_{3}\right)$, $\operatorname{IR}\left(\right.$ film) $\nu_{\max }: 3052,2925,2854,1759,1654,1466,1373,1318,1203,1149,1102,1033,919,722 \mathrm{~cm}^{-1}$. ${ }^{1} \mathrm{H}$ NMR $\left(500 \mathrm{MHz}, \mathrm{CDCl}_{3}\right) \delta: 0.88(3 \mathrm{H}, \mathrm{t}, J=6.7 \mathrm{~Hz}), 1.25-1.50(46 \mathrm{H}, \mathrm{m}), 1.41(3 \mathrm{H}, \mathrm{d}, J=6.8 \mathrm{~Hz})$, $1.92(2 \mathrm{H}, \mathrm{m}), 2.26(2 \mathrm{H}, \mathrm{t}, J=6.7 \mathrm{~Hz}), 3.39(6 \mathrm{H}, \mathrm{s}), 3.46(2 \mathrm{H}, \mathrm{m}), 3.98(2 \mathrm{H}, \mathrm{m}), 4.67(2 \mathrm{H}, \mathrm{d}, J=6.8 \mathrm{~Hz})$, $4.84(2 \mathrm{H}, \mathrm{d}, J=6.8 \mathrm{~Hz}), 5.00(1 \mathrm{H}, \mathrm{qd}, J=6.8,1.5 \mathrm{~Hz}), 6.99(1 \mathrm{H}, \mathrm{d}, J=1.5 \mathrm{~Hz}) \mathrm{ppm} .{ }^{13} \mathrm{C} \mathrm{NMR}(125$ $\left.\mathrm{MHz}, \mathrm{CDCl}_{3}\right) \delta: 14.13,19.21,22.69,25.16,25.52,27.38,28.44,29.18,29.31,29.35,29.52,29.61,29.64$, 29.80, 31.22, 31.91, 55.71, 77.41, 79.60, 81.49, 96.67, 134.31, 148.86, 173.93 pm. HREIMS Calcd for $\mathrm{C}_{38} \mathrm{H}_{69} \mathrm{O}_{6}[\mathrm{M}-\mathrm{OMe}]^{+}$621.5094, found 621.5086.

Solamin (1). $\mathrm{BF}_{3} \cdot \mathrm{Et}_{2} \mathrm{O}(0.02 \mathrm{~mL})$ was added to a solution of $\mathbf{1 8}(5 \mathrm{mg}, 0.076 \mathrm{mmol})$ in dimethyl sulfide $(0.4 \mathrm{ml})$ at $0^{\circ} \mathrm{C}$. After the mixture was stirred for $10 \mathrm{~min}$ at this temperature, the reaction was quenched with saturated aqueous $\mathrm{NaHCO}_{3}(1 \mathrm{ml})$, and the mixture was extracted with diethyl ether. The organic phase was washed with brine, dried over anhydrous $\mathrm{MgSO}_{4}$, filtered, and concentrated. The residue was purified with preparative TLC (hexane/AcOEt $=1: 2)$ to afford $\mathbf{1}(3.0 \mathrm{mg}, 72 \%)$ as a colorless waxy solid. M.p. 67-69 ${ }^{\circ} \mathrm{C},[\alpha]^{25}+22\left(c 0.20, \mathrm{CH}_{3} \mathrm{OH}\right), \operatorname{IR}(\mathrm{KBr}) v_{\max }: 3438,3052,2918,2850,1737,1653,1468$, 1374, 1203, 1115, 1082, 1028, 961, 890, $721 \mathrm{~cm}^{-1} .{ }^{1} \mathrm{H}$ NMR $\left(500 \mathrm{MHz}, \mathrm{CDCl}_{3}\right) \delta: 0.88(3 \mathrm{H}, \mathrm{t}, J=7.1$ Hz), 1.21-1.58 (44H, m), 1.41 (3H, d, J = 6.8 Hz), 1.68 (2H, m), 2.00 (2H, m), 2.25-2.31 (4H, m), 3.40 $(2 \mathrm{H}, \mathrm{m}), 3.80(2 \mathrm{H}, \mathrm{m}), 5.00(1 \mathrm{H}, \mathrm{dd}, J=6.8,1.4 \mathrm{~Hz}), 6.99(1 \mathrm{H}, \mathrm{d}, J=1.4 \mathrm{~Hz}) \mathrm{ppm} .{ }^{13} \mathrm{C} \mathrm{NMR}(125 \mathrm{MHz}$, $\mathrm{CDCl}_{3}$ ) $\delta: 14.09,19.22,22.68,25.19,25.61,27.43,28.73,29.18,29.30,29.35,29.50,29.59,29.62,29.64$, 29.72, 31.92, 33.56, 74.04, 77.36, 81.83, 134.40, 148.79, 173.84 pm. HRFABMS Calcd for $\mathrm{C}_{35} \mathrm{H}_{64} \mathrm{O}_{5} \mathrm{Na}$ $[\mathrm{M}+\mathrm{Na}]^{+}$587.4651, found 587.4650.

$(1 ', S, 2$ ' $R, 5 S, 5$ ' $S, 13$ 'R)-3-\{13'-Methoxymethoxy-13'-[5"-(1'"'-methoxymethoxytridecyl)tetrahydr-o furan-2"-yl]tetradec-8'en-10'-ynyl\}-5-methyl-2,5-dihydrofuran-2-one (19). The procedure was the same as that used for the preparation of 17. Compound 19 (97 mg, 84\%) was prepared from 10 (75 $\mathrm{mg}$, $0.45 \mathrm{mmol}$ ) and 9 (90 mg, $0.21 \mathrm{mmol})$ as a colorless oil. IR(film) $v_{\max }: 3052,2925,2853,1758,1654$, 1466, 1318, 1204, 1150, 1102, 1041, 955, $918 \mathrm{~cm}^{-1}$. ${ }^{1} \mathrm{H}$ NMR $\left(500 \mathrm{MHz}, \mathrm{CDCl}_{3}\right) \delta: 0.88(3 \mathrm{H}, \mathrm{t}, J=7.0$ $\mathrm{Hz}), 1.25-1.95(39 \mathrm{H}, \mathrm{m}), 2.07(2 \mathrm{H}, \mathrm{m}), 2.26(2 \mathrm{H}, \mathrm{t}, J=7.0 \mathrm{~Hz}), 2.48-2.74(2 \mathrm{H}, \mathrm{m}), 3.39(3 \mathrm{H}, \mathrm{s}), 3.40(1 \mathrm{H}$, 
s), $3.41(2 \mathrm{H}, \mathrm{s}), 3.51(1 \mathrm{H}, \mathrm{m}), 3.68(1 \mathrm{H}, \mathrm{m}), 3.92(1 \mathrm{H}, \mathrm{m}), 4.08(1 \mathrm{H}, \mathrm{m}), 4.67(1 \mathrm{H}, \mathrm{d}, J=6.8 \mathrm{~Hz})$, 4.73-4.78 (3H, m), $5.00(1 \mathrm{H}, \mathrm{qd}, J=7.0,1.5 \mathrm{~Hz}), 5.42(0.67 \mathrm{H}, \mathrm{dd}, J=15.5,1.5 \mathrm{~Hz}), 5.81(0.67 \mathrm{H}, \mathrm{m})$, $6.03(0.67 \mathrm{H}, \mathrm{dt}, J=15.5,7.0 \mathrm{~Hz}), 6.99(1 \mathrm{H}, \mathrm{d}, J=1.5 \mathrm{~Hz}) \mathrm{ppm} .{ }^{13} \mathrm{C} \mathrm{NMR}\left(125 \mathrm{MHz}, \mathrm{CDCl}_{3}\right) \delta: 14.09$, $19.18,22.39,22.56,22.65,25.12,25.14,25.40,27.33,27.46,27.73,28.69,28.85,28.90,29.06,29.15$, $29.32,29.59,29.60,29.63,29.78,30.10,31.20,31.88,32.87,55.67,66.64,77.80,77.89,79.76,80.33$, 80.58, 82.01, 84.85, 96.39, 96.67, 109.16, 109.68, 134.22, 142.94, 143.71, 148.87, 173.84 pm. HREIMS Calcd for $\mathrm{C}_{39} \mathrm{H}_{67} \mathrm{O}_{7}[\mathrm{M}]^{+}$646.4809, found 647.4793.

$(1 ", S, 2$ 2" $R, 5 S, 5$ 'S,13'R)-3-\{13'-Methoxymethoxy-13'-[5"-(1'"'-methoxymethoxytridecyl)tetrahydrofuran-2"-yl]tetradecyl\}-5-methyl-2,5-dihydrofuran-2-one (20). The procedure was the same as that used for the preparation of 18. Compound 20 (85 mg, 85\%) was prepared from 19 (96 mg, $0.15 \mathrm{mmol})$ as a colorless oil. $[\alpha]_{\mathrm{D}}^{19}+4.5\left(c 0.87, \mathrm{CHCl}_{3}\right), \mathrm{IR}(\mathrm{film}) v_{\max }: 3052,2924,2853,1760,1654,1466,1318$, 1149, 1102, 1078, 1041, $918 \mathrm{~cm}^{-1} .{ }^{1} \mathrm{H}$ NMR (500 MHz, $\left.\mathrm{CDCl}_{3}\right) \delta: 0.88(3 \mathrm{H}, \mathrm{t}, J=6.8 \mathrm{~Hz}), 1.25-1.50$ $(46 \mathrm{H}, \mathrm{m}), 1.41(3 \mathrm{H}, \mathrm{d}, J=7.0 \mathrm{~Hz}), 1.86(2 \mathrm{H}, \mathrm{m}), 2.26(2 \mathrm{H}, \mathrm{t}, J=7.5 \mathrm{~Hz}), 3.39(6 \mathrm{H}, \mathrm{s}), 3.50(2 \mathrm{H}, \mathrm{m}), 3.89$ $(2 \mathrm{H}, \mathrm{m}), 4.67(2 \mathrm{H}, \mathrm{d}, J=7.0 \mathrm{~Hz}), 4.83(2 \mathrm{H}, \mathrm{d}, J=6.5 \mathrm{~Hz}), 5.00(1 \mathrm{H}, \mathrm{qd}, J=7.0,1.5 \mathrm{~Hz}), 6.99(1 \mathrm{H}, \mathrm{d}, J=$ $1.5 \mathrm{~Hz})$ ppm. ${ }^{13} \mathrm{C}$ NMR (125 MHz, $\left.\mathrm{CDCl}_{3}\right) \delta: 14.10,19.21,22.67,25.17,25.44,27.39,27.60,29.25$, $29.34,29.52,29.61,29.65,29.66,29.81,31.23,31.91,55.68,77.38,79.81,81.83,96.70,134.33,148.82$, 173.88 pm. HREIMS Calcd for $\mathrm{C}_{38} \mathrm{H}_{69} \mathrm{O}_{6}[\mathrm{M}-\mathrm{OMe}]^{+}$621.5094, found 621.5090.

cis-Solamin (2). The procedure was the same as that used for the preparation of 1. Compound 2 (36 mg, $76 \%$ ) was prepared from 20 (55 mg, $0.84 \mathrm{mmol})$ as a colorless waxy solid. M.p. 70-72 ${ }^{\circ} \mathrm{C},[\alpha]^{19} \mathrm{D}+21(c$ 0.34, $\left.\mathrm{CH}_{3} \mathrm{OH}\right), \mathrm{IR}(\mathrm{KBr}) v_{\max }: 3422,3052,2918,2849,1739,1653,1469,1081,1029,969,721 \mathrm{~cm}^{-1} .{ }^{1} \mathrm{H}$ NMR (500 MHz, $\left.\mathrm{CDCl}_{3}\right) \delta: 0.88(3 \mathrm{H}, \mathrm{t}, J=6.8 \mathrm{~Hz}), 1.21-1.58(44 \mathrm{H}, \mathrm{m}), 1.41(3 \mathrm{H}, \mathrm{d}, J=6.8 \mathrm{~Hz}), 1.75$ $(2 \mathrm{H}, \mathrm{m}), 1.94(2 \mathrm{H}, \mathrm{m}), 2.26(2 \mathrm{H}, \mathrm{t}, J=7.0 \mathrm{~Hz}), 2.55(2 \mathrm{H}$, brs. $-\mathrm{OH}), 3.42(2 \mathrm{H}, \mathrm{m}), 3.82(2 \mathrm{H}, \mathrm{m}), 5.00$ $(1 \mathrm{H}, \mathrm{dd}, J=6.8,1.5 \mathrm{~Hz}), 6.99(1 \mathrm{H}, \mathrm{d}, J=1.5 \mathrm{~Hz}) \mathrm{ppm} .{ }^{13} \mathrm{C} \mathrm{NMR}\left(125 \mathrm{MHz}, \mathrm{CDCl}_{3}\right) \delta: 14.10,19.19$, 22.66, 25.14, 25.69, 27.36, 28.09, 29.05, 29.15, 29.21, 29.27, 29.33, 29.39, 29.47, 29.57, 29.60, 29.62, 29.64, 29.65, 29.66, 29.68, 31.89, 34.07, 74.33, 77.39, 82.69, 134.30, 148.84, 173.89 pm. HREIMS Calcd for $\mathrm{C}_{35} \mathrm{H}_{64} \mathrm{O}_{5}[\mathrm{M}]^{+}$564.4753, found 564.4720.

\section{REFERENCES AND NOTES}

1. For recent reviews, see: (a) H. Makabe, H. Konno, and H. Miyoshi, Curr. Drug. Discovery Technol., 2008, 5, 213. (b) N. Li, Z. Shi, Y. Tang, J. Chen, and X. Li, Beilstein J. Org. Chem., 4, 2008, published 05 December. (c) Makabe, H. Biosci. Biotechnol. Biochem., 2007, 71, 2367. (d) J. L. McLaughlin, J. Nat. Prod., 2008, 71, 1311. (d) A. Bermejo, B. Figadère, M.-C. Zafra-Polo, I. 
Barrachina, E. Estoneroll, and D. Cortes, Nat. Prod. Rep., 2005, 22, 269.

2. Recent synthesis of annonaceous acetogenins: (a) C. L. Morris, Y. Hu, G. D. Head, L. J. Brown, W. G. Whittingham, and R. C. D. Brown, J. Org. Chem., 2009, 74, 981. (b) N. D. Griggs and A. J. Phillips, Org. Lett., 2008, 10, 4955. (c) S. Takahashi, Y. Hongo, Y. Tsukagoshi, and H. Koshino, Org. Lett., 2008, 10, 4223. (d) S. Furuhata, Y. Hattori, M. Okajima, H. Konno, M. Abe, H. Miyoshi, T. Goto, and H. Makabe, Tetrahedron, 2008, 64, 7695. (e) C. W. Huh and W. R. Roush, Org. Lett., 2008, 10, 3371. (f) Y. Hattori, S. Furuhata, M. Okajima, H. Konno, M. Abe, H. Miyoshi, T. Goto, and H. Makabe, Org. Lett., 2008, 10, 717.

3. S. H. Myint, D. Cortes, A. Laurens, R. Hocquemiller, M. Leboeuf, A. Cavé, J. Cotte, and A. -M. Quero, Phytochemistry, 1991, 30, 3335.

4. C. Gleye, P. Duret, A. Laurens, R. Hocquemiller, and A. Cavé, J. Nat. Prod. 1998, 61, 576.

5. Synthesis of solamin: (a) S. C. Sinha and E. Keinan, J. Am. Chem. Soc., 1993, 115, 4891. (b) B. M. Trost and Z. Shi, J. Am. Chem. Soc., 1994, 116, 7459. (c) H. Makabe, A. Tanaka, and T. Oritani, J. Chem. Soc. Perkin Trans 1, 1994, 1975. (d) W. Kuriyama, K. Ishigami, and T. Kitahara, Heterocycles, 1999, 50, 981. (e) S. Raghavan, S. G. Subramanian, and K. A. Tony, Tetrahedron Lett., 2008, 49, 1601.

6. Synthesis of cis-solamin: (a) H. Makabe, Y. Hattori, A. Tanaka, and T. Oritani, Org. Lett., 2002, 4, 1083. (b) H. Makabe, Y. Hattori, Y. Kimura, H. Konno, M. Abe, H. Miyoshi, A. Tanaka, and T. Oritani, Tetrahedron, 2004, 60, 10651. (c) A. R. L. Cecil and R. C. D. Brown, Org. Lett., 2002, 4, 3715. (d) A. R. L. Cecil, Y. Hu, M. J. Vicent, R. Duncan, and R. C. D. Brown, J. Org. Chem., 2004, 69, 3368. (e) H. Göksel and C. B. Stark, Org. Lett., 2006, 8, 3433. (f) H. Konno, Y. Okuno, H. Makabe, K. Nosaka, A. Onishi, Y. Abe, A. Sugimoto, and K. Akaji, Tetrahedron Lett., 2008, 49, 782.

7. H. Makabe, A. Miyawaki, R. Takahashi, Y. Hattori, H. Konno, M. Abe, and H. Miyoshi, Tetrahedron Lett, 2004, 45, 973.

8. Y. Hattori, Y. Kimura, A. Moroda, H. Konno, M. Abe, H. Miyoshi, T. Goto, and H. Makabe, Chem. Asian. J. 2006, 1, 894.

9. J. D. White, T. C. Somers, and G. N. Reddy, J. Org. Chem., 1992, 57, 4991. 